\title{
nature/methods Focus on Mapping the Brain
}

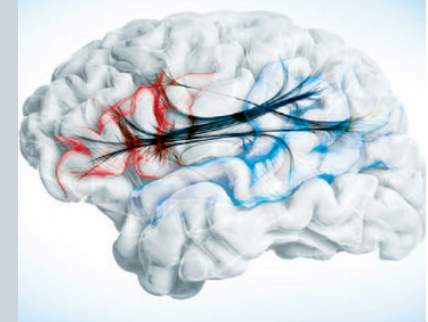

Visualization of functional connectivity in the human cerebral cortex based on magnetic resonance imaging data. Brain image by Joachim Böttger and Daniel Margulies (Max Planck Institute for Human Cognitive and Brain Sciences, Leipzig, Germany) with compositing help by Tobias S. Hoffmann. Cover composition by Erin Dewalt.

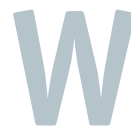

e are entering a new era in neuroscience in which technological development will allow us to obtain full anatomical, high-resolution renderings of entire brain circuits and to map the activity of ever larger cellular populations as an animal performs specific behaviors. Assembling anatomical, molecular and functional maps has the potential to greatly advance our understanding of how brains work.

In this Focus, experts outline the technologies needed to obtain these maps and discuss what will be needed beyond them to understand brain function.

In a Historical Perspective, Cornelia Bargmann and Eve Marder discuss what has been learned from invertebrate circuits whose connectivity patterns are known and what will be needed beyond anatomical maps to understand brain function in other organisms. In a Commentary, Jeff Lichtman and Joshua Morgan express their views about why obtaining detailed, high-resolution structural maps should be an essential part of this endeavor. To understand the deluge of data these maps will engender once generated, Olaf Sporns argues in another Commentary that data representation and modeling will be critical.

Other experts discuss the newest technologies available for obtaining brain maps. Moritz Helmstaedter presents the state of the art and current challenges of electron microscopy-based circuit reconstruction. In three papers, the potential of using light to unveil the function and anatomy of brain circuits is presented. Karl Deisseroth and Kwanghun Chung discuss their newly developed method named CLARITY for rendering mammalian brains permeable to visible photons and molecules. Pavel Osten and Troy Margrie review light-microscopy methods available for large-scale anatomical tracing and discuss ways to integrate molecular identity, activity recording and anatomical information. In a Resource, Josh Sanes and colleagues present improved tools for mapping the mouse brain using the Brainbow technology. Finally, Michael Milham, Stan Colcombe and their colleagues review methods for functional and anatomical analysis of human brains at the macroscale.

We are pleased to acknowledge the financial support of Carl Zeiss Microscopy, Hamamatsu Corporation, LaVision BioTec, TissueVision, Inc. and Chroma Technology Corp. Nature Methods carries sole responsibility for all editorial content and peer review. Erika Pastrana

\section{CONTENTS}

483 From the connectome to brain function

C I Bargmann \& E Marder

491 Making sense of brain network data

O Sporns

494 Why not connectomics?

JL Morgan \&

$J$ W Lichtman

501 Cellular-resolution connectomics: challenges of dense neural circuit reconstruction M Helmstaedter

508 CLARITY for mapping the nervous system $K$ Chung \& K Deisseroth

515 Mapping brain circuitry with a light microscope

P Osten \& $T W$ Margrie

524 Imaging human connectomes at the macroscale

$R$ C Craddock, S Jbabdi, C-G Yan, J T Vogelstein, $F X$ Castellanos, A Di Martino, C Kelly, K Heberlein, $S$ Colcombe \& MP Milham

540 Improved tools for the Brainbow toolbox

Dawen Cai, K B Cohen, T Luo, J W Lichtman \& $J R$ Sanes
Editor, Nature Methods Daniel Evanko

Focus Editor Erika Pastrana

Publisher Stephanie Diment

Senior Copy Editor Irene Kaganman
Copy Editor Odelia Ghodsizadeh Managing Production Editor Renee Lucas

Production Editors Brandy Cafarella,
Carol Evangelista, Ivy Robles

Sponsorship David Bagshaw, Yvette Smith, Reya Silao

Marketing Nazly De La Rosa 